\title{
NOTES ON A PAPER BY SANOV
}

RUTH REBEKKA STRUIK

Introduction. In this paper a proof is given of some results obtained by Sanov (see [2]). Some identities are used which are of interest in themselves.

Let $F=\{u, v\}$ be a free group generated by $u, v$. Let

$$
(x, y)=x^{-1} y^{-1} x y
$$

for $x, y F$. If $S, T$ are subgroups of $F$, let

$$
(S, T)=\{(s, t), s \in S, t \in T\} .
$$

Let

$$
F(k)=\left\{x^{k}, x \in F\right\}, \quad F_{1}=F, \quad F_{k}=\left(F_{k-1}, F\right) .
$$

Let

$$
(u, v, 1)=(u, v) ; \quad(u, v, n)=((u, v, n-1), v) .
$$

Then Sanov [2] proved that

$$
\left(u, v, \alpha p^{\alpha}-1\right)^{p^{\beta-\alpha}} \in F\left(p^{\beta}\right) F_{\alpha p^{\alpha}+1}, \quad \beta, \alpha=1,2, \cdots
$$

where $p$ is an arbitrary prime.

In this paper a proof simpler than Sanov's is given for the case $p=2$ and $p=3$, with $\alpha=1$, and $\beta$ arbitrary. It also gives a result if instead of $p$ being a prime, $p$ is divisible by 2 or 3 . Identities (4), (6), (9) and (10) below are of interest in themselves.

Theorem 1. Let $F=\{u, v\}$ be a free group generated by $u$, v. Let $k=2 n$. Then

$$
(u, v)^{k / 2} \in F(k) F_{3} \quad k=2,4,6, \cdots
$$

and, in particular, if $k=2^{\beta}$, then

$$
(u, v)^{2^{\beta-1}} \in F\left(2^{\beta}\right) F_{3} \quad \beta=1,2, \cdots .
$$

Comment. (3) is (1) for $\alpha=1, p=2$.

ProOF. By induction on $k$

$$
\begin{aligned}
d & =(u, v)\left(v, u^{2}\right) \cdots\left(v^{j-1}, u^{j}\right)\left(u^{i}, v^{j}\right) \cdots\left(u^{k-1}, v^{k-1}\right) \\
& =\left(u^{-1} v^{-1}\right)^{k}\left(v u v^{-1}\right)^{k} v^{k} \in F(k), \quad k=2,3, \cdots
\end{aligned}
$$

Received by the editors December 5, 1956. 
can be proved.

Let $R=\{x, y\}$ be a free associative ring generated by $x, y$ and let $u, v$ correspond to $1+x, 1+y$ respectively (see [1] or Theorem M1 in $[3])$. Then (4) will correspond to

$$
1+P_{2}(x, y)+\cdots
$$

where $P_{2}$ is a polynomial in $x, y$ of degree 2 . Since $\left(u^{i}, v^{j}\right)$ corresponds to $1+i j[x, y]+\cdots$ where $[x, y]=x y-y x$,

$$
\begin{aligned}
P_{2}(x, y)= & (1-(1 \cdot 2-2 \cdot 2)+\cdots+(-(j-1) \cdot j+j \cdot j)+\cdots \\
& +(k-1)(k-1))[x, y] \\
= & (1+2+\cdots+j+\cdots+(k-1))[x, y] \\
= & ((k-1) k / 2)[x, y] .
\end{aligned}
$$

Hence $d \equiv(u, v)^{k(k-1) / 2} \bmod F_{3}$, i.e.,

$$
(u, v)^{k(k-1) / 2} \in F(k) F_{3}
$$

since $d \in F(k)$. If $k$ is odd, (5) is a trivial statement. If $k$ is even, then for $k=2 n$,

$$
k(k-1) / 2=n(2 n-1) \equiv-n \equiv n \bmod 2 n
$$

i.e., for $k$ even, $(u, v)^{k / 2} \in F(k) F_{3}$. q.e.d.

Comment. Theorem 1 can also be proved using the identity

$$
v^{-k} u^{-k}(u v)^{k}=\left(v, u^{k-1}\right)\left(\left(v, u^{k-1}\right), v^{k-1}\right) \cdots
$$

$$
\left(v, u_{j}\right)\left(\left(v, u_{j}\right), v_{j}\right) \cdots(v, u)((v, u), v) . k=2,3, \cdots .
$$

Theorem 2. Let $F=\{u, v\}$ be a free group generated by $u$, v. Let $k=3 n$. Then

$$
((u, v), v)^{k / 3} \in F(k) F_{4}
$$

and, in particular, if $k=3^{\beta}$, then

$$
((u, v), v)^{3^{\beta-1}} \in F\left(3^{\beta}\right) F_{4} .
$$

Comment. Note that (8) is (1) with $\alpha=1, p=3$.

Proof.

$$
\begin{aligned}
f & =\left(v^{-1} u^{-1}\right)^{k}\left(u v u v^{-1} u^{-1}\right)^{k}\left(u v u^{-2}\right)^{-k} u^{k} \\
& =\left(v^{-1} u^{-1}\right)^{k-2} v^{-1} u^{k-2}\left(v u^{-1}\right)^{k-2} v u^{k-2} \\
& =(v, u)\left(u, v^{2}\right)\left(v^{2}, u^{2}\right) \cdots\left(v^{i}, u^{j}\right)\left(u^{j}, v^{j+1}\right) \cdots\left(u^{k-2}, v^{k-1}\right) \\
& \cdot\left(v^{k-2}, u\right)\left(u, v^{k-3}\right) \cdots\left(v^{k-j-1}, u^{j}\right)\left(u^{j}, v^{k-j-2}\right) \cdots\left(u^{k-3}, v\right)\left(v, u^{k-2}\right) \\
& \in F(k) \cap F(k-2) \quad k=3,4, \cdots
\end{aligned}
$$


can be proved.

$$
\begin{aligned}
& \left(v^{-1} u^{-1}\right)^{k-2} v^{-1} \\
& \quad=(v, u)\left(u, v^{2}\right) \cdots\left(v^{j}, u^{j}\right)\left(u^{j}, v^{i+1}\right) \cdots\left(u^{k-2}, v^{k-1}\right) v^{-(k-1)} u^{-(k-2)}
\end{aligned}
$$

which can be proved by induction is useful in proving (10). (11) is a variation of $(4)$.

$$
v^{-(k-1)}\left(v^{-j}, u^{j}\right)\left(u^{j}, v^{-j-1}\right) v^{k-1}=\left(v^{k-j-1}, u^{j}\right)\left(u^{j}, v^{k-j-2}\right)
$$

is also useful.

That $f \in F_{2}$ is obvious. To show $f \in F_{3}$, map $F=\{u, v\}$ into the free associative ring, $R=\{x, y\}$ with $u, v$ corresponding to $1+x$ and $1+y$ respectively. Investigate $Q_{n}(x, y)$ where

$$
1+Q_{2}(x, y)+Q_{3}(x, y)+\cdots
$$

is the element of $R$ onto which $f$ is mapped, and $Q_{m}(x, y)$ is a polynomial of degree $m$. A somewhat laborious computation shows that

$$
\begin{aligned}
& Q_{2}(x, y)=0, \\
& Q_{3}(x, y)=[[x, y], y]\left(\begin{array}{l}
k \\
3
\end{array}\right) \text { where }\left(\begin{array}{l}
k \\
3
\end{array}\right)=\frac{k(k-1)(k-2)}{6} \\
& k=3,4, \cdots .
\end{aligned}
$$

In computing $Q_{n}(x, y)$ the following lemma is of use:

LEMMA.

$$
\begin{aligned}
& \left(u^{m}, v^{n}\right) \equiv(u, v)^{m n}((u, v), u)_{2}^{n(m)}((u, v), v)_{2}^{m(n)} \bmod F_{4} \\
& \left(v^{m}, u^{n}\right)=(u, v)^{-m n}((u, v), u)_{2}^{-m(n)}((u, v), v)_{2}^{-n(m)} \bmod F_{4}
\end{aligned}
$$

where

$$
\left(\begin{array}{l}
m \\
2
\end{array}\right)=\frac{m(m-1)}{2} .
$$

Proof of lemma is by induction and uses the identities

$$
\begin{aligned}
& (x y, z)=(y,(z, x))(x, z)(y, z), \\
& (x, y z)=(x, z)(x, y)((x, y), z)
\end{aligned}
$$

and the fact that (see [1])

$$
\begin{aligned}
\left(x^{r},\left(y^{s}, x^{t}\right)\right) & \equiv(x,(y, x))^{r s t} \equiv((x, y), x)^{r s t} \bmod F_{4}, \\
((x, y), x) & \equiv((y, x), x)^{-1} \bmod F_{4}
\end{aligned}
$$

where $r, s, t$ are arbitrary integers. 
In computing $Q_{3}(x, y)$, the identities

$$
\left(\begin{array}{l}
n \\
3
\end{array}\right)+\left(\begin{array}{l}
n \\
2
\end{array}\right)=\left(\begin{array}{c}
n+1 \\
3
\end{array}\right) \text { and }\left(\begin{array}{l}
n \\
2
\end{array}\right)+n=\left(\begin{array}{c}
n+1 \\
2
\end{array}\right)
$$

are also useful. Using (13) and investigating

$$
\left(\begin{array}{l}
k \\
3
\end{array}\right) \text { for } k=6 n, 6 n+1, \cdots, 6 n+5 \text {, }
$$

one finds that the only nontrivial cases are

$$
\begin{aligned}
& k=3 n, \quad\left(\begin{array}{l}
k \\
3
\end{array}\right) \equiv k / 3 \bmod k, \\
& k=3 n+2 \quad\left(\begin{array}{l}
k \\
3
\end{array}\right) \equiv(k-2) / 3 \bmod k-2,
\end{aligned}
$$

and this shows that for $k=3 n$,

$$
f \equiv((u, v), v)^{k / 3} \bmod F_{4}
$$

and hence for $k=3 n$

$$
((u, v), v)^{k / 3} \in F(k) F_{4} \quad \text { q.e.d. }
$$

\section{BIBLIOGRAPHY}

1. W. Magnus, Über Beziehungen zwischen höheren Kommutatoren, J. Reine Angew. Math. vol. 177 (1937) pp. 105-115.

2. I. N. Sanov, O Nekotorï Sisteme Sootnoshenii v Periodicheskikh Gruppakh s Periodom Ctepeniu Protogo Chisla (On a certain system of relations in periodic groups with period a power of a prime number), Bull. Acad. Sci. URSS. Ser. Math. 15 (1951) pp. 477-502.

3. R. R. Struik, On associative products of groups, Trans. Amer. Math. Soc. vol. 81 (1956) pp. 425-452.

Drexel Institute of TeChNology 